S. Shirai

Nagoya Math. J.

Vol. 75 (1979), 133-143

\title{
ON THE CENTRAL IDEAL CLASS GROUP OF CYCLOTOMIC FIELDS
}

\author{
SUSUMU SHIRAI
}

\section{Introduction}

Let $\boldsymbol{Q}$ be the rational number field, $K / \boldsymbol{Q}$ be a finite Galois extension with the Galois group $G$, and let $C_{K}$ be the ideal class group of $K$ in the wider sense. We consider $C_{K}$ as a $G$-module. Denote by $I$ the augmentation ideal of the group ring of $G$ over the ring of rational integers. Then $C_{K} / I\left(C_{K}\right)$ is called the central ideal class group of $K$, which is the maximal factor group of $C_{K}$ on which $G$ acts trivially. A. Fröhlich [3,4] rationally determined the central ideal class group of a complete ${ }^{1)}$ Abelian field over $\boldsymbol{Q}$ whose degree is some power of a prime. The proof is based on Theorems 3 and 4 of Fröhlich [2]. D. Garbanati [6] recently gave an algorithm which will produce the $\ell$-invariants of the central ideal class group of an Abelian extension over $\boldsymbol{Q}$ for each prime $\ell$ dividing its order.

In the present paper we determine the central ideal class group of a cyclotomic field over $\boldsymbol{Q}$ in terms of generators and relations by refining upon the methods used in $[3,4](\S 3$, Theorem 5$)$. The proof is based on Theorem 32 of our preceding paper [10], which is a generalization of Fröhlich [2, Theorem 3] to the case of a cyclotomic field over $\boldsymbol{Q}$.

\section{Notation}

Throughout this paper the following notation will be used.

$\boldsymbol{Q}$ the field of rational numbers as in Introduction.

$Z$ the ring of rational integers on which a finite group acts trivially.

Received August 4, 1978.

1) Cf. Fröhlich $\left[3\right.$, p. 212] and $\left[4\right.$, pp. 73-77]. When $[K: Q]=\ell^{\alpha}$, this implies that $K_{0}^{*}=K$ or $K^{*}=K$ according as $\ell=2, K$ real or otherwise, where $K_{0}^{*}$ is the maximal real, unramified, Abelian 2-extension of $K$ which is still Abelian over $Q$, and $K^{*}$ is the maximal, unramified, Abelian $\ell$-extension of $K$ which is still Abelian over $\boldsymbol{Q}$. 
$Z_{n} \quad$ the cyclic group of order $n$.

$\langle A\rangle \quad$ the subgroup generated by $A$ when $A$ is a subset in a group. $(a, b)$ the commutator $a b a^{-1} b^{-1}$ of $a$ and $b$ when $a, b$ are elements in a group.

$(A, B)$ the subgroup generated by the commutators $(a, b)$ of all $a \in A$, $b \in B$ when $A, B$ are subsets in a group.

$A \times B \quad$ the direct product of $A$ by $B$ when $A, B$ are groups.

$G(K / k)$ the Galois group of $K$ over $k$.

$(, K / k)$ the norm residue symbol for $K / k$ when $K / k$ is a local Abelian extension.

$\left(\frac{, K}{p}\right)$ the norm residue symbol for $K$ when $K$ is a finite Abelian ex-

$C_{K} / I\left(C_{K}\right)$ the central ideal class group of $K$ defined in Introduction when $K$ is a finite Galois extension over $\boldsymbol{Q}$.

$\psi(n) \quad$ the Euler's function, i.e. the number of positive integers not exceeding $n$ which are relatively prime to $n$.

$(m, n) \quad$ the G.C.D. of $m$ and $n$ when $m, n$ are rational integers.

Moreover we will use the results and notation of the preceding paper [10].

\section{§1. The Schur multiplicator of a finite Abelian group}

The structure of the Schur multiplicator $H^{-3}(G, Z)$ of a finite Abelian group $G$ is well-known (cf. [7], [8], [9]). In this section we describe $H^{-3}(G, Z)$ in terms of generators and relations.

LEMMA 1. If $G=Z_{n_{1}} \times \cdots \times Z_{n_{r}}$, then

$$
\left|H^{-3}(G, Z)\right|=\prod_{1 \leqq i<j \leqq r}\left(n_{i}, n_{j}\right) .
$$

Proof. We proceed by induction on $r$. For any Abelian group $A$, and any integer $q$, we denote by $A(q)$ the subgroup comprising all those elements $a$ of $A$ such that $a^{q}=1$. Then it follows from R. C. Lyndon [7, Lemma 8.2] that

$$
\begin{aligned}
H^{-3}(G, Z) \cong H^{3}(G, Z) & \\
\cong & H^{3}\left(Z_{n_{r}}, Z\right) \times \prod_{0<k<3} H^{k}\left(Z_{n_{1}} \times \cdots \times Z_{n_{r-1}}, Z\right)\left(n_{r}\right) \\
& \times H^{3}\left(Z_{n_{1}} \times \cdots \times Z_{n_{r-1}}, Z\right) \\
\cong & \left(Z_{n_{1}} \times \cdots \times \mathbf{Z}_{n_{r-1}}\right)\left(n_{r}\right) \times H^{3}\left(Z_{n_{1}} \times \cdots \times Z_{n_{r-1}}, Z\right),
\end{aligned}
$$

because $H^{1}(G, Z)=1$ and $H^{2}(G, Z) \cong G$ for any finite Abelian group $G$. 
Thus by induction hypothesis,

$$
\begin{aligned}
\left|H^{-3}(G, Z)\right| & =\prod_{1 \leqq i<j \leqq r-1}\left(n_{i}, n_{j}\right) \cdot\left(n_{1}, n_{r}\right) \cdots\left(n_{r-1}, n_{r}\right) \\
& =\prod_{1 \leqq i<j \leqq r}\left(n_{i}, n_{j}\right) .
\end{aligned}
$$

Lemma 2. Let $G=Z_{n_{1}} \times \cdots \times Z_{n_{r}}$, and let

$$
1 \longrightarrow(\Omega, \Omega) \longrightarrow \Omega \stackrel{f}{\longrightarrow} G \longrightarrow 1
$$

be an exact sequence in which $\Omega$ is a finite nilpotent group of class two such that $(\Omega, \Omega) \cong H^{-3}(G, Z)$. Denote by $\omega_{i}$ an element of $\Omega$ such that $f\left(\omega_{i}\right)$ is a generator of $Z_{n_{i}}(\subset G)$ for $i=1, \cdots, r$. Then $(\Omega, \Omega)$ is generated by $\left(\begin{array}{l}r \\ 2\end{array}\right)$ elements

$$
\left(\omega_{i}, \omega_{j}\right), \quad 1 \leqq i<j \leqq r,
$$

and completely determined by the relations

$$
\begin{cases}\left(\omega_{i}, \omega_{j}\right)\left(\omega_{k}, \omega_{l}\right)=\left(\omega_{k}, \omega_{l}\right)\left(\omega_{i}, \omega_{j}\right), & \text { all } i, j, k, l, \\ \left(\omega_{i}, \omega_{j}\right)^{\left(n_{i}, n_{j}\right)}=1, & 1 \leqq i<j \leqq r .\end{cases}
$$

Proof. Since $\Omega$ is of class two, it is obvious that the elements $\left(\omega_{i}, \omega_{j}\right)$, $1 \leqq i<j \leqq r$ generate $(\Omega, \Omega)$, and satisfy the above relations (1). Furthermore the order of $(\Omega, \Omega)$ is $\prod_{1 \leqq i<j \leqq r}\left(n_{i}, n_{j}\right)$ by Lemma 1 . Conversely the group which is generated by $\left(\begin{array}{l}r \\ 2\end{array}\right)$ elements and satisfies the above relations (1) is an Abelian group of order $\prod_{1 \leqq i<j \leqq r}\left(n_{i}, n_{j}\right)$. Hence $(\Omega, \Omega)$ is completely described by the relations (1).

Q.E.D.

\section{§2. Inertia groups}

Let $p$ be a rational prime, $\boldsymbol{Q}_{p}$ be the $p$-adic number field, $T / \boldsymbol{Q}_{p}$ be a finite unramified extension, $\zeta$ be a primitive $p^{\nu}$-th root of unity, and let $K=T(\zeta)$. Denote by $\hat{K}$ a central extension of $K / \boldsymbol{Q}_{p}$ such that the $p$ exponent $\mu\left(\hat{K} / \boldsymbol{Q}_{p}\right)$ of the Galois conductor ${ }^{2)}$ of $\hat{K} / \boldsymbol{Q}_{p}$ does not exceed $\nu$.

Lemma 3. Let $p=2$, and let

$$
\sigma=\left(2, K / \boldsymbol{Q}_{2}\right)^{-1}, \quad \tau^{*}=\left(-1, K / \boldsymbol{Q}_{2}\right), \quad \tau=\left(5, K / \boldsymbol{Q}_{2}\right) .
$$

Denote by $\tilde{\sigma}, \tilde{\tau}^{*}$, and $\tilde{\tau}$ any extensions of $\sigma, \tau^{*}$, and $\tau$ to $\hat{K}$, respectively.

2) See $[10, \S 1]$. 
Then the inertia group of $\hat{K} / K$ is generated by the elements $\left(\tilde{\tau}, \tilde{\tau}^{*}\right),(\tilde{\tau}, \tilde{\sigma})$, $\left(\tilde{\tau}^{*}, \tilde{\sigma}\right)$.

Proof. Let $F$ be the inertia field of $\hat{K} / K$, and let $D$ be the fixed field of $\left\langle\left(\tilde{\tau}, \tilde{\tau}^{*}\right),(\tilde{\tau}, \tilde{\sigma}),\left(\tilde{\tau}^{*}, \tilde{\sigma}\right)\right\rangle$. Since $G\left(\hat{K} / \boldsymbol{Q}_{2}\right)$ is of class two and $\left\{\tilde{\sigma}, \tilde{\tau}^{*}, \tilde{\tau}\right\}$ is a system of generators of $G\left(\hat{K} / \boldsymbol{Q}_{2}\right)$, the commutator group of $G\left(\hat{K} / \boldsymbol{Q}_{2}\right)$ is generated by the elements $\left(\tilde{\tau}, \tilde{\tau}^{*}\right),(\tilde{\tau}, \tilde{\sigma}),\left(\tilde{\tau}^{*}, \tilde{\sigma}\right)$. Thus $D / \boldsymbol{Q}_{2}$ is the maximal Abelian extension contained in $\hat{K}$. Hence $D \supset F$, because $F / Q_{2}$ is an Abelian extension.

To prove the converse let $T^{\prime}$ be the inertia field of $D / Q_{2}$. Since $\mu\left(D / Q_{2}\right) \leqq \mu\left(\hat{K} / \boldsymbol{Q}_{2}\right) \leqq \nu$ by [10, Lemma 3], it follows from local class field theory that $G\left(D / T^{\prime}\right)$ is a homomorphic image of the group of prime residue classes $\bmod 2^{\nu}$. We have $\left[D: T^{\prime}\right] \leqq \psi\left(2^{\nu}\right)=2^{\nu-1}$, and hence $D=T^{\prime} K$, because of $T^{\prime} \cap K=T,\left[T^{\prime} K: T^{\prime}\right]=[K: T]=2^{\nu-1}$. We conclude that $D / K$ is unramified, which implies $F \supset D$.

Q.E.D.

By the same procedure as the proof of Lemma 3, we obtain

Lemma 4. Let $p \neq 2, g$ be a primitive root $\bmod p^{\nu}$, and let

$$
\sigma=\left(p, K / \boldsymbol{Q}_{p}\right)^{-1}, \quad \tau=\left(g, K / \boldsymbol{Q}_{p}\right) .
$$

Denote by $\tilde{\sigma}$ and $\tilde{\tau}$ any extensions of $\sigma$ and $\tau$ to, $\hat{K}$, respectively. Then the inertia group of $\hat{K} / K$ is generated by the single element $(\tilde{\tau}, \tilde{\sigma})$.

\section{§3. The central ideal class group of cyclotomic fields}

Let $m=2^{\nu^{y}} p_{1}^{\nu_{1}} \cdots p_{r}^{\nu_{r}}$ be a positive integer, $K$ be the $m$-th cyclotomic field over $\boldsymbol{Q}$, and let $\hat{K}$ be the central class field $\bmod m p_{\infty}$ in the sense of $[10, \S 3]$, where $p_{\infty}$ is the real prime divisor of $\boldsymbol{Q}$. Then $\hat{K}$ is a central extension of $K / Q$, and hence it is a nilpotency class two extension over $\boldsymbol{Q}$. Moreover it follows from the definition of the central class field $\bmod m$ that any rational prime not contained in $m p_{\infty}$ is unramified in $\hat{K}$. We have already proved in [10, Theorem 32] that if $(m, 16) \neq 8$, then

$$
(G(\hat{K} / Q), G(\hat{K} / Q))=G(\hat{K} / K) \cong H^{-3}(G(K / Q), Z) .
$$

For use of this result we distinguish the following three cases:
(a) $\nu=0$,
(b) $\nu=2$,
(c) $\nu \geqq 4$.

In the present paper we will prove our main Theorem for (a) and state the corresponding results for (b) and (c). 
Assume $\nu=0$. Let $g_{i}$ be a primitive $\operatorname{root} \bmod p_{i}^{\nu i}$, and let

$$
\sigma_{i}=\left(\frac{p_{i}, K}{p_{i}}\right)^{-1}, \quad \tau_{i}=\left(\frac{g_{i}, K}{p_{i}}\right), \quad i=1, \cdots, r
$$

Since $G(K / Q)$ is isomorphic to the group of prime residue classes $\bmod m$, $G(K / Q) \cong Z_{\psi\left(p_{1}^{\left.\nu_{1}\right)}\right.} \times \cdots \times Z_{\psi\left(p_{r}^{\nu}\right)}$, and $\left\{\tau_{1}, \cdots, \tau_{r}\right\}$ is a system of generators of $G(K / Q)$. For each $i$, we choose elements $\tilde{\sigma}_{i}$ and $\tilde{\tau}_{i}$ in the decomposition group of a prime factor $\mathfrak{\beta}_{i}$ of $p_{i}$ in $\hat{K}$, which under the natural homomorphism of $G(\hat{K} / \boldsymbol{Q})$ onto $G(K / Q)$ are mapped onto $\sigma_{i}$ and $\tau_{i}$, respectively. Since $G(\hat{K} / K)$ is contained in the center of $G(\hat{K} / Q)$, the inertia group of $\mathfrak{P}_{i}$ over $K$ does not depend on the choice of $\mathfrak{P}_{i}$ over $p_{i}$, and it is generated by the element $\left(\tilde{\tau}_{i}, \tilde{\sigma}_{i}\right)$, as we can see by Lemma 4 .

According to Lemma 2 and $(2), G(\hat{K} / K)$ is generated by $\left(\begin{array}{l}r \\ 2\end{array}\right)$ elements

$$
\left(\tilde{\tau}_{i}, \tilde{\tau}_{j}\right), \quad 1 \leqq i<j \leqq r,
$$

and completely determined by the relations

$$
\begin{gathered}
\left(\tilde{\tau}_{i}, \tilde{\tau}_{j}\right)\left(\tilde{\tau}_{k}, \tilde{\tau}_{l}\right)=\left(\tilde{\tau}_{k}, \tilde{\tau}_{l}\right)\left(\tilde{\tau}_{i}, \tilde{\tau}_{j}\right), \quad \text { all } i, j, k, l, \\
\left(\tilde{\tau}_{i}, \tilde{\tau}_{j}\right)^{\left(\psi\left(p_{i}^{\nu} i\right), \psi\left(p_{j}^{\nu j)}\right)\right.}=1, \quad 1 \leqq i<j \leqq r .
\end{gathered}
$$

Let $C_{K}$ be the ideal class group ${ }^{3)}$ of $K$, and let $U$ be the Abelian extension of $K$ corresponding to $I\left(C_{K}\right)$ in the sense of class field theory. Then $U$ is the maximal central extension of $K / Q$ which is unramified over $K$, and is contained in $\hat{K}$, as we can see by going back to the definition of the central class field mod $m$. We conclude that $U$ is the subfield of $\hat{K}$ corresponding to $\left\langle\left(\tilde{\tau}_{1}, \tilde{\sigma}_{1}\right), \cdots,\left(\tilde{\tau}_{r}, \tilde{\sigma}_{r}\right)\right\rangle$ in the sense of Galois theory. Hence

$$
C_{K} / I\left(C_{K}\right) \cong G(U / K) \cong G(\hat{K} \mid K) /\left\langle\left(\tilde{\tau}_{1}, \tilde{\sigma}_{1}\right), \cdots,\left(\tilde{\tau}_{r}, \tilde{\sigma}_{r}\right)\right\rangle
$$

We next express $\left(\tilde{\tau}_{i}, \tilde{\sigma}_{i}\right)$ in terms of $\tilde{\tau}_{1}, \cdots, \tilde{\tau}_{r}$. Define the symbols ${ }^{4}$ $[j, i],[0, i]^{*},[0, i]$ by putting

$$
\left\{\begin{array}{lc}
p_{i} \equiv g_{j}^{[j, i]} \bmod p_{j}^{\nu j}, \quad i=0,1, \cdots, r, j=1, \cdots, r, \\
p_{i} \equiv(-1)^{[0, i]^{*} 5^{[0, i]} \bmod 2^{\nu},} \quad i=1, \cdots, r, \\
{[i, i]=0,} & i=1, \cdots, r,
\end{array}\right.
$$

3) In this case the ideal class groups in the narrow and the wider sense coincide, because no real prime divisor exists in $K$.

4) Cf. Fröhlich [2, pp. 237-238]. 
where $p_{0}=2$, namely, $[j, i]$ is the index of $p_{i}$ for the modulus $p_{j}^{\nu_{j}}$ relative to the primitive root $g_{j}$, and $[0, i]^{*},[0, i]$ are the indices of $p_{i}$ for the modulus $2^{\nu}$ relative to the basis $\{-1,5\}$. Then we have

$$
\sigma_{i}=\prod_{j=1}^{r} \tau_{j}^{[j, i]} \quad \text { for } i=1, \cdots, r,
$$

because of $\prod_{\text {al1 } p}\left(\frac{p_{i}, K}{p}\right)=1$, the product formula in class field theory. Therefore

$$
\begin{aligned}
\left(\tilde{\tau}_{i}, \tilde{\sigma}_{i}\right) & =\tilde{\tau}_{i} \tilde{\sigma}_{i} \tilde{\tau}_{i}^{-1} \tilde{\sigma}_{i}^{-1}=\left(\tilde{\tau}_{i}, \prod_{j=1}^{r} \tilde{\tau}_{j}^{[j, i]}\right) \\
& =\prod_{j=1}^{r}\left(\tilde{\tau}_{i}, \tilde{\tau}_{j}\right)^{[j, i]},
\end{aligned}
$$

because $G(\hat{K} / K)$ is contained in the center of $G(\hat{K} / Q)$ and $G(\hat{K} / Q)$ is of class two. Thus ${ }^{5)}$ we have proved the following main

THEOREM 5. Let $m=2^{\nu} p_{1}^{\nu_{1}} \cdots p_{r}^{\nu_{r}}$ be a positive integer, $K$ be the $m$-th cyclotomic field over $\boldsymbol{Q}$, and let $C_{K} / I\left(C_{K}\right)$ be the central ideal class group of $K$. Then:

(a) $\nu=0 . C_{K} / I\left(C_{R}\right)$ is generated by $\left(\begin{array}{l}r \\ 2\end{array}\right)$ elements $x_{i j}, 1 \leqq i<j \leqq r$, and completely determined by the relations

$$
\begin{array}{ll}
x_{i j} x_{k l}=x_{k l} x_{i j}, & \text { all } i, j, k, l, \\
\prod_{j=1}^{r} x_{i j}^{[j, i]}=1, & i=1, \cdots, r, \\
x_{i j}^{\left(\psi\left(p_{i}^{v i}\right),\left(p_{j}^{\nu j)}\right)\right.}=1, & 1 \leqq i<j \leqq r,
\end{array}
$$

with the convention $x_{j i}=x_{i j}^{-1}$.

(b) $\nu=2 . C_{K} / I\left(C_{K}\right)$ is generated by $\left(\begin{array}{c}r+1 \\ 2\end{array}\right)$ elements $x_{i j}, 0 \leqq i<j \leqq r$, and completely determined by the relations

$$
\begin{array}{ll}
x_{i j} x_{k l}=x_{k l} x_{i j}, & \text { all } i, j, k, l, \\
\prod_{j=1}^{r} x_{0 j}^{[j, 0]}=1, & \\
x_{0 i}^{-[0, i]^{*}} \prod_{j=1}^{r} x_{i j}^{[j, i]}=1, & i=1, \cdots, r, \\
x_{0 i}^{2}=1, & i=1, \cdots, r,
\end{array}
$$

5) As regards computation in the cases (b) and (c), cf. [11, §3]. See also Fröhlich [3, Theorem 2] and [4, Theorem 3]. 


$$
x_{i j}^{\left(\psi\left(p_{i}^{\nu i}\right), \psi\left(p_{j}^{\nu j}\right)\right)}=1, \quad 1 \leqq i<j \leqq r,
$$

with the convention $x_{j i}=x_{i j}^{-1}$.

(c) $\nu \geqq 4 . \quad C_{K} / I\left(C_{K}\right)$ is generated by $\left(\begin{array}{c}r+2 \\ 2\end{array}\right)-1$ elements $x_{i j},-1 \leqq i$ $<j \leqq r,(i, j) \neq(-1,0)$, and completely determined by the relations

$$
\begin{array}{ll}
x_{i j} x_{k l}=x_{k l} x_{i j}, & \text { all } i, j, k, l, \\
\prod_{j=1}^{r} x_{i j}^{[j, 0]}=1, & i=-1,0, \\
x_{-1 i}^{-[0, i]} x_{0 i}^{-[0, i]^{*}} \prod_{j=1}^{r} x_{i j}^{[j, i]}=1, & i=1, \cdots, r \\
x_{-1 i}^{\left(2 \nu-2, \psi\left(p_{i}^{\nu i}\right)\right)}=1, & i=1, \cdots, r, \\
x_{0 i}^{2}=1, & i=1, \cdots, r, \\
x_{i j}^{\left(\psi\left(p_{i}^{\nu}\right), \psi\left(p_{j}^{\nu j}\right)\right)}=1, & 1 \leqq i<j \leqq r,
\end{array}
$$

with the convention $x_{j i}=x_{i j}^{-1}$, where $[j, i],[0, i]^{*},[0, i]$ are the indices defined by (3).

\section{§4. Applications}

Y. Furuta [5, Theorem 4] proved the following result: Let $\ell$ be any rational prime and $m$ be a rational integer. Assume that the number of different prime divisors $p$ of $m$ such that $p \equiv 1 \bmod \ell$ is equal to or greater than 8 (this number should be replaced by 9 , only when $\ell=2$ and $m$ $\not \equiv 0 \bmod 4)$. Then the class number of the $m$-th cyclotomic field is always divisible by $\ell$ and moreover the $m$-th cyclotomic field admits an infinite unramified $\ell$-extension.

The first half of this result can be sharpen as follows.

TheOREM $6^{6)}$. Let $m=2^{y_{1} \nu_{1}^{1}} \cdots p_{r}^{\nu_{r}}$ be a positive integer, $K$ be the $m$-th cyclotomic field over $Q, \rho_{\ell}$ be the $\ell$-rank of $C_{K} / I\left(C_{K}\right)$, and let $t$ be the number of different primes $p_{i}$ of $m$ such that $p_{i} \equiv 1 \bmod \ell$. Then:

(a) $\nu=0$. $\quad \rho_{\ell} \geqq \frac{1}{2} t(t-3)$.

In particular $\rho_{2} \geqq \frac{1}{2} r(r-3)$.

(b) $\nu=2$. $\quad \rho_{\ell} \geqq \frac{1}{2} t(t-3), \quad \ell \neq 2$,

$\rho_{2} \geqq \frac{1}{2}(r+1)(r-2)$.

(c) $\quad \nu \geqq 4$. $\quad \rho_{\ell} \geqq \frac{1}{2} t(t-3), \quad \ell \neq 2$, $\rho_{2} \geqq \frac{1}{2}\left(r^{2}+r-4\right)$.

Proof. (a) Suppose the primes $p_{i}$ to be so numbered that $p_{i} \equiv 1$ $\bmod \ell$ for $i=1, \cdots, t$. Set $m^{\prime}=p_{1}^{\nu_{1}} \cdots p_{t}^{\nu_{t}}$, and denote by $C^{\prime}$ the central

6) Cf. Fröhlich [3, Lemmas 2 and 3] and [4, Lemmas 4 and 5]. 
ideal class group of the $m^{\prime}$-th cyclotomic field over $\boldsymbol{Q}$. By virtue of Theorem 5, (a), $C^{\prime}$ is generated by $\left(\begin{array}{l}t \\ 2\end{array}\right)$ elements $y_{i j}, 1 \leqq i<j \leqq t$, and completely determined by the relations

$$
\begin{array}{ll}
y_{i j} y_{k l}=y_{k l} y_{i j}, & \text { all } i, j, k, l, \\
\prod_{j=1}^{t} y_{i j}^{[j, i]}=1, & i=1, \cdots, t, \\
y_{i j}^{\left(p_{i}^{\nu}\left(p_{i}^{\nu}\right), \psi\left(p_{j}^{\nu j}\right)\right)}=1, & 1 \leqq i<j \leqq t,
\end{array}
$$

where $y_{j i}=y_{i j}^{-1}$. We define a homomorphism $C_{K} / I\left(C_{K}\right) \rightarrow C^{\prime}$ by putting $x_{i j} \rightarrow y_{i j}$ for $1 \leqq i<j \leqq t, x_{i j} \rightarrow 1$ otherwise. Then the homomorphism is epimorphic. Hence denoting the $\ell$-rank of $C^{\prime}$ by $\rho_{\ell}^{\prime}$, we have

$$
\rho_{\ell} \geqq \rho_{\ell}^{\prime} \text {. }
$$

It follows from the assumption that

$$
\left(\psi\left(p_{i}^{\nu_{i}}\right), \psi\left(p_{j}^{y_{j}}\right)\right) \equiv 0 \bmod \ell \quad \text { for } 1 \leqq i<j \leqq t .
$$

Noting the convention $y_{j i}=y_{i j}^{-1}$, we denote by $A$ the matrix of coefficients in the additively written equations (4) on generators $y_{i j}$ and by $r(A)$ its rank as a matrix in $G F(\ell)$. Since $A$ is $a\left(t, \frac{1}{2} t(t-1)\right)$ matrix, we have $r(A) \leqq t$. Hence

$$
\rho_{\ell}^{\prime}=\frac{1}{2} t(t-1)-r(A) \geqq \frac{1}{2} t(t-3) .
$$

Q.E.D.

CoRollaRY 7. Let $m=2^{y} p_{1}^{\nu_{1}} \cdots p_{r}^{\nu_{r}}$ be a positive integer, $h$ be the class number of the $m$-th cyclotomic field over $\boldsymbol{Q}$, and let $t$ be the number of different primes $p_{i}$ of $m$ such that $p_{i} \equiv 1 \bmod \ell$ for an odd prime $\ell$. Then:

(a) $\nu=0$. If $r \geqq 4$, then $2^{2} \mid h$.

If $t \geqq 4$, then $2^{2} \ell^{2} \mid h$.

(b) $\nu=2$. If $r \geqq 3$, then $2^{2} \mid h$.

If $t \geqq 4$, then $2^{5} \ell^{2} \mid h$.

(c) $\nu \geqq 4$. If $r \geqq 2$, then $2 \mid h$.

If $t \geqq 4$, then $2^{8} \ell^{2} \mid h$.

In any case $h$ is divisible by 4 if $r \geqq 4$, and by $2^{2} \ell^{2}$ if $t \geqq 4$.

Finally we state a result concerning the invariants of the central ideal class group of cyclotomic fields. The following Lemma can be 
easily verified ${ }^{7}$.

Lemma 8. For any prime $p$ and any integer a prime to $p$, let

$$
\begin{aligned}
& o\left(p^{\nu}, a\right)=\text { the order of } a \bmod p^{\nu} \text { for } \nu \geqq 1 \\
& q(p, a)=\text { the highest exponent of } p \text { dividing } a^{p-1}-1 .
\end{aligned}
$$

Then we have: (i) If $p \neq 2$ and $\alpha=q(p, a)$, then

$$
\begin{aligned}
& o(p, a)=o\left(p^{2}, a\right)=\cdots=o\left(p^{\alpha}, a\right), \\
& o\left(p^{\alpha+i}, a\right)=p^{i} o(p, a) \quad \text { for } i \geqq 1 .
\end{aligned}
$$

(ii) $p=2$. If $\alpha=q(2, a)>1$, i.e. $a \equiv 1 \bmod 4$, then

$$
\begin{aligned}
& o(2, a)=o\left(2^{2}, a\right)=\cdots=o\left(2^{\alpha}, a\right)=1, \\
& o\left(2^{\alpha+i}, a\right)=2^{i} \quad \text { for } i \geqq 1,
\end{aligned}
$$

and if $\alpha=1$, i.e. $a \equiv 3 \bmod 4$, then

$$
\begin{aligned}
& o\left(2^{2}, a\right)=o\left(2^{3}, a\right)=\cdots=o\left(2^{\beta}, a\right)=2, \\
& o\left(2^{\beta+i}, a\right)=2^{i+1} \quad \text { for } i \geqq 1,
\end{aligned}
$$

where $\beta=$ the highest exponent of 2 dividing $a^{2}-1$. Note $\beta \geqq 3$, which implies that the group of prime residue classes $\bmod 2^{\nu}$ is not cyclic when $\nu \geqq 3$.

Denote by $B$ the matrix of coefficients in the additively written equations on generators $x_{i j}$ in Theorem 5, (a), noting $x_{j i}=x_{i j}^{-1}$. Then $B$ is a $\left(\frac{1}{2} r(r+1), \frac{1}{2} r(r-1)\right)$ matrix. Let $e_{i}$ be the elementary divisors of $B$ in the domain of rational integers such that $e_{1}\left|e_{2}\right| \cdots \mid e_{s}, e_{i}>0, i=1, \cdots, s$. Then $C_{K} / I\left(C_{K}\right)$ can be written in the form, as a product of cyclic groups,

$$
C_{K} / I\left(C_{K}\right) \cong Z_{e_{1}} \times \cdots \times Z_{e_{s}} .
$$

Each $e_{i}$ can be computed by the following rule: Let $D_{i}(B)$ be the G.C.D. of all $i$-th minors in $\operatorname{det} B$. Then

$$
D_{i}(B)=e_{1} e_{2} \cdots e_{i}, \quad 1 \leqq i \leqq s .
$$

Hence

$$
\begin{aligned}
e_{1} & =\text { the G.C.D. of all entries of } B \\
& =\text { G.C.D. }\left\{\left(\psi\left(p_{i}^{\nu_{i}}\right), \psi\left(p_{j}^{\nu^{j}}\right),[j, i],[i, j]\right) \mid 1 \leqq i<j \leqq r\right\} \\
& =\text { G.C.D. }\left\{\left(\frac{\psi\left(p_{i}^{\nu_{i}}\right)}{o\left(p_{i}^{\nu_{i}}, p_{j}\right)}, \frac{\psi\left(p_{j}^{\nu_{j}}\right)}{o\left(p_{j^{j}}^{j}, p_{i}\right)}\right) \mid 1 \leqq i<j \leqq r\right\} .
\end{aligned}
$$

7) See also L. E. Dickson [1, Chapter VII]. 
Thus by virtue of Lemma 8 we obtain

TheOREM 9. Let $m=p_{1}^{\nu_{1}} \cdots p_{r}^{\nu_{r}}, p_{1}, \cdots, p_{r}$ distinct odd primes, and let $C_{K} / I\left(C_{K}\right)$ be the central ideal class group of the $m$-th cyclotomic field over $\boldsymbol{Q}$. Then the first elementary divisor $e_{1}$ of $C_{K} / I\left(C_{K}\right)$ in (5) becomes constant for all $\nu_{i}$ sufficiently large. In fact if we put $\alpha_{i j}=q\left(p_{i}, p_{j}\right)$ and take $\nu_{i}>\max \left\{\alpha_{i j} \mid j=1, \cdots, r\right\}$ for $i=1, \cdots, r$, then

$$
e_{1}=\text { G.C.D. }\left\{\left(\frac{\psi\left(p_{i}^{\alpha_{i j}}\right)}{o\left(p_{i}, p_{j}\right)}, \frac{\psi\left(p_{j}^{\alpha}{ }_{j i}\right)}{o\left(p_{j}, p_{i}\right)}\right) \mid 1 \leqq i<j \leqq r\right\}
$$

ExAMPLE. For $m=5^{\nu_{1}} 11^{\nu_{2}}, \nu_{1} \geqq 2, \nu_{2} \geqq 2$, we have $C_{K} / I\left(C_{K}\right) \cong Z_{2}$.

Remark. Let $K$ be a finite Galois extension over $\boldsymbol{Q}, f(K)$ be its Galois conductor in the sense of $[10, \S 2]$, and let $m$ be a rational module such that $f(K) \mid m$. We denote by $\hat{K}_{m}$ the central class field $\bmod m$ and by $K_{m}^{*}$ the genus field $\bmod m$ of $K / \boldsymbol{Q}$ in the sense of $[10, \S 3]$. Then it follows from [10, Theorem 31] that if all first ramification groups of $K / Q$ are cyclic, then

$$
G\left(\hat{K}_{m} / K_{m}^{*}\right) \cong H^{-3}(G(K / Q), Z)
$$

Thus the method leading up to main Theorem 5 can be applicable to determine the central ideal class group of Abelian extensions over $\boldsymbol{Q}$ whose first ramification groups are cyclic, because it is based on Lemma 2 and (2).

\section{REFERENCES}

[1] L. E. Dickson, History of the Theory of Numbers, vol. 1, Washington 1919.

[2] A. Fröhlich, On fields of class two, Proc. London Math. Soc. (3), 4 (1954), 235256.

[ 3 ] — , On the absolute class-group of Abelian fields, J. London Math. Soc., 29 (1954), 211-217.

[4] - On the absolute class-group of Abelian fields (II), J. London Math. Soc., 30 (1955), 72-80.

[5] Y. Furuta, On class field towers and the rank of ideal class groups, Nagoya Math. J., 48 (1972), 147-157.

[6] D. Garbanati, Invariants of the ideal class group and the Hasse norm theorem, $J$. reine angew. Math., 297 (1978), 159-171.

[ 7 ] R. C. Lyndon, The cohomology theory of group extensions, Duke Math. J., 15 (1948), 271-292.

[ 8 ] I. Schur, Über die Darstellung der endlichen Gruppen durch gebrochene lineare Substitutionen, J. reine angew. Math., 127 (1904), 20-50.

[9] I. Schur, Untersuchungen über die Darstellung der endlichen Gruppen durch 
gebrochene lineare Substitutionen, ibid., 132 (1907), 85-137.

[10] S. Shirai, On the central class field $\bmod \mathfrak{m}$ of Galois extensions of an algebraic number field, Nagoya Math. J., 71 (1978), 61-85.

[11] — On Galois groups of class two extensions over the rational number field, Nagoya Math. J., 75 (1979), 121-131.

Toyama Medical and Pharmaceutical University 
